\title{
Precise Measurement of the Solar Neutrino Day/Night and Seasonal Variation in Super-Kamiokande-I
}

\author{
The Super-Kamiokande Collaboration
}

M.B. Smy ${ }^{5}$, Y. Ashie ${ }^{1}$, S. Fukuda ${ }^{1}$, Y. Fukuda ${ }^{1}$, K. Ishihara ${ }^{1}$, Y. Itow ${ }^{1}$, Y. Koshio ${ }^{1}$, A. Minamino ${ }^{1}$, M. Miura ${ }^{1}$, S. Moriyama ${ }^{1}$, M. Nakahata ${ }^{1}$, T. Namba ${ }^{1}$, R. Nambu ${ }^{1}$, Y. Obayashi ${ }^{1}$, N. Sakurai ${ }^{1}$, M. Shiozawa ${ }^{1}$, Y. Suzuki ${ }^{1}$, H. Takeuchi ${ }^{1}$, Y. Takeuchi ${ }^{1}$, S. Yamada ${ }^{1}$, M. Ishitsuka ${ }^{2}$, T. Kajita ${ }^{2}$, K. Kaneyuki ${ }^{2}$, S. Nakayama ${ }^{2}$, A. Okada ${ }^{2}$, T. Ooyabu ${ }^{2}$, C. Saji ${ }^{2}$, S. Desai ${ }^{3}$, M. Earl ${ }^{3}$, E. Kearns ${ }^{3}$, M.D. Messier ${ }^{3, *}$, J.L. Stone ${ }^{3}$, L.R. Sulak ${ }^{3}$, C.W. Walter ${ }^{3}$, W. Wang ${ }^{3}$, M. Goldhaber ${ }^{4}$, T. Barszczak ${ }^{5}$, D. Casper ${ }^{5}$, W. Gajewski ${ }^{5}$, W.R. Kropp ${ }^{5}$, S. Mine ${ }^{5}$, D.W. Liu ${ }^{5}$, H.W. Sobel ${ }^{5}$, M.R. Vagins ${ }^{5}$, A. Gago ${ }^{6}$, K.S. Ganezer ${ }^{6}$, J. Hill ${ }^{6}$, W.E. Keig ${ }^{6}$, J.Y. Kim ${ }^{7}$, I.T. Lim ${ }^{7}$, R.W. Ellsworth ${ }^{8}$, S. Tasaka ${ }^{9}$, A. Kibayashi ${ }^{10}$, J.G. Learned ${ }^{10}$, S. Matsuno ${ }^{10}$, D. Takemori ${ }^{10}$, Y. Hayato ${ }^{11}$,

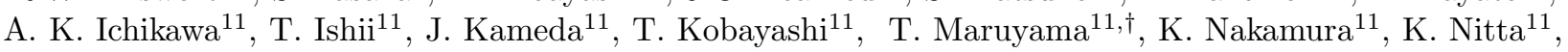
Y. Oyama ${ }^{11}$, M. Sakuda ${ }^{11}$, Y. Totsuka ${ }^{11}$, M. Yoshida ${ }^{11}$, T. Iwashita ${ }^{12}$, A.T. Suzuki ${ }^{12}$, T. Inagaki ${ }^{13}$, I. Kato ${ }^{13}$, T. Nakaya ${ }^{13}$, K. Nishikawa ${ }^{13}$, T.J. Haines ${ }^{14,5}$, S. Dazeley ${ }^{15}$, S. Hatakeyama ${ }^{15}$, R. Svoboda ${ }^{15}$, E. Blaufuss ${ }^{16}$, J.A. Goodman ${ }^{16}$, G. Guillian ${ }^{16}$, G.W. Sullivan ${ }^{16}$, D. Turcan $^{16}$, K. Scholberg ${ }^{17}$, A. Habig ${ }^{18}$, M. Ackermann ${ }^{19}$, C.K. Jung ${ }^{19}$, T. Kato ${ }^{19}$, K. Kobayashi ${ }^{19}$, K. Martens ${ }^{19, \ddagger}$, M. Malek ${ }^{19}$, C. Mauger ${ }^{19}$, C. McGrew ${ }^{19}$, E. Sharkey ${ }^{19}$, B. Viren ${ }^{19,4}$ C. Yanagisawa ${ }^{19}$, T. Toshito ${ }^{20}$, C. Mitsuda ${ }^{21}$, K. Miyano ${ }^{21}$, T. Shibata ${ }^{21}$, Y. Kajiyama ${ }^{22}$, Y. Nagashima ${ }^{22}$, M. Takita ${ }^{22}$, H.I. Kim ${ }^{23}$, S.B. Kim${ }^{23}$, J. Yoo ${ }^{23}$, H. Okazawa ${ }^{24}$, T. Ishizuka ${ }^{25}$, Y. Choi ${ }^{26}$,

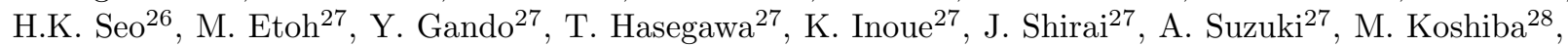
Y. Hatakeyama ${ }^{29}$, Y. Ichikawa ${ }^{29}$, M. Koike ${ }^{29}$, K. Nishijima ${ }^{29}$, H. Ishino ${ }^{30}$, M. Morii ${ }^{30}$, R. Nishimura ${ }^{30}$, Y. Watanabe ${ }^{30}$, D. Kielczewska ${ }^{31,5}$, J. Zalipska ${ }^{31}$, H.G. Berns ${ }^{32}$, S.C. Boyd ${ }^{32}$, A.L. Stachyra ${ }^{32}$, R.J. Wilkes ${ }^{32}$

1 Kamioka Observatory, Institute for Cosmic Ray Research, University of Tokyo, Kamioka, Gifu, 506-1205, Japan

${ }^{2}$ Research Center for Cosmic Neutrinos, Institute for Cosmic Ray Research, University of Tokyo, Kashiwa, Chiba 277-8582, Japan 3 Department of Physics, Boston University, Boston, MA 02215, USA

4 Physics Department, Brookhaven National Laboratory, Upton, NY 11973, USA

5 Department of Physics and Astronomy, University of California, Irvine, Irvine, CA 92697-4575, USA

6 Department of Physics, California State University, Dominguez Hills, Carson, CA 90747, USA

7 Department of Physics, Chonnam National University, Kwangju 500-757, Korea

8 Department of Physics, George Mason University, Fairfax, VA 22030, USA

9 Department of Physics, Gifu University, Gifu, Gifu 501-1193, Japan

10 Department of Physics and Astronomy, University of Hawaii, Honolulu, HI 96822, USA

11 Institute of Particle and Nuclear Studies, High Energy Accelerator Research Organization (KEK), Tsukuba, Ibaraki 305-0801, Japan

12 Department of Physics, Kobe University, Kobe, Hyogo 657-8501, Japan

13 Department of Physics, Kyoto University, Kyoto 606-8502, Japan

14 Physics Division, P-23, Los Alamos National Laboratory, Los Alamos, NM 87544, USA

15 Department of Physics and Astronomy, Louisiana State University, Baton Rouge, LA 70803, USA

16 Department of Physics, University of Maryland, College Park, MD 20742, USA

17 Department of Physics, Massachusetts Institute of Technology, Cambridge, MA 02139, USA

18 Department of Physics, University of Minnesota, Duluth, MN 55812-2496, USA

19 Department of Physics and Astronomy, State University of New York, Stony Brook, NY 11794-3800, USA

20 Department of Physics, Nagoya University, Nagoya, Aichi 464-8602, Japan

21 Department of Physics, Niigata University, Niigata, Niigata 950-2181, Japan

22 Department of Physics, Osaka University, Toyonaka, Osaka 560-0043, Japan

23 Department of Physics, Seoul National University, Seoul 151-742, Korea

24 International and Cultural Studies, Shizuoka Seika College, Yaizu, Shizuoka, 425-8611, Japan

25 Department of Systems Engineering, Shizuoka University, Hamamatsu, Shizuoka 432-8561, Japan

26 Department of Physics, Sungkyunkwan University, Suwon 440-746, Korea

27 Research Center for Neutrino Science, Tohoku University, Sendai, Miyagi 980-8578, Japan

28 The University of Tokyo, Tokyo 113-0033, Japan

29 Department of Physics, Tokai University, Hiratsuka, Kanagawa 259-1292, Japan

30 Department of Physics, Tokyo Institute for Technology, Meguro, Tokyo 152-8551, Japan

31 Institute of Experimental Physics, Warsaw University, 00-681 Warsaw, Poland

32 Department of Physics, University of Washington, Seattle, WA 98195-1560, USA

The time variation of the elastic scattering rate of solar neutrinos with electrons in SuperKamiokande-I was fit to the variations expected from active two-neutrino oscillations. The best fit in the Large Mixing Angle solution has a mixing angle of $\tan ^{2} \theta=0.55$ and a mass squared difference of $\Delta m^{2}=6.3 \times 10^{-5} \mathrm{eV}^{2}$ between the two neutrino mass eigenstates. The fitted day/night asymmetry of $-1.8 \pm 1.6$ (stat) $)_{-1.2}^{+1.3}$ (syst)\% has improved statistical precision over previous measurements and agrees well with the expected asymmetry of $-2.1 \%$. 
The combined analysis of all solar neutrino experiments 1, 2] gives firm evidence for neutrino oscillations. All data are well described using just two neutrino mass eigenstates and imply a mass squared difference between $\Delta m^{2}=3 \times 10^{-5} \mathrm{eV}^{2}$ and $\Delta m^{2}=1.9 \times 10^{-4} \mathrm{eV}^{2}$ and $\mathrm{a}$ mixing angle between $\tan ^{2} \theta=0.25$ and $\tan ^{2} \theta=0.65[\underline{3}]$. This region of parameter space is referred to as the Large Mixing Angle solution (LMA). The rate and spectrum of reactor anti-neutrino interactions in the KamLAND experiment [4] are also well reproduced for these mixing angles and some of these $\Delta m^{2}$. Over the $\Delta m^{2}$ range of the LMA, solar ${ }^{8} \mathrm{~B}$ neutrinos are $\approx 100 \%$ resonantly converted into the second mass eigenstate by the large matter density inside the sun [5]. Therefore, the survival probability into $\nu_{e}$ is $\approx \sin ^{2} \theta$. However, due to the presence of the earth's matter density, the oscillation probability at an experimental site on earth into $\nu_{e}$ differs from $\sin ^{2} \theta$ during the night. Since our experiment is primarily sensitive to $\nu_{e}$ 's, this induces an apparent dependence of the measured neutrino interaction rate on the solar zenith angle (often a regeneration of $\nu_{e}$ 's during the night). We employ a maximum likelihood fit to the expected solar zenith angle dependence on the neutrino interaction rate. Herein, the statistical uncertainty is reduced by $25 \%$ compared to our previous measurement of the day/night asymmetry [2, 3] which consists of two flux measurements in two separate data samples (day and night). It would require almost three more years of running time to obtain a similar uncertainty reduction with the previous method.

Super-Kamiokande (SK) is a 50,000 ton water Cherenkov detector described in detail elsewhere [6]. SK measures the energy, direction, and time of the recoil electron from elastic scattering of solar neutrinos with electrons by detection of the emitted Cherenkov light. Super-Kamiokande started taking data in April, 1996. In this report, we analyze the full SK-I low energy data set consisting of 1496 live days (May 31 ${ }^{\text {st }}, 1996$ through July $\left.15^{\text {th }}, 2000\right)$.

The solar neutrino interactions are separated from background events by taking advantage of the strong forward peak of the elastic scattering cross section. The arrival time of each solar neutrino candidate defines a solar direction. Using this direction, we calculate the angle $\theta_{\text {sun }}$ between the reconstructed recoil electron direction and the solar direction. The data sample is divided into $N_{\text {bin }}=21$ energy bins: 18 energy bins of $0.5 \mathrm{MeV}$ between 5 and $14 \mathrm{MeV}$, two energy bins of $1 \mathrm{MeV}$ between 14 and $16 \mathrm{MeV}$, and one bin between 16 and $20 \mathrm{MeV}$. We use two types of probability density functions: $p\left(\cos \theta_{\text {sun }}, E\right)$ describes the angular shape expected for solar $\nu_{e}$ 's of energy $E$ (signal events) and $u_{i}\left(\cos \theta_{\text {sun }}\right)$ is the background shape in energy bin $i$. Each of the $n_{i}$ events in energy bin $i$ is assigned the background factor $b_{i \kappa}=u_{i}\left(\cos \theta_{i \kappa}\right)$

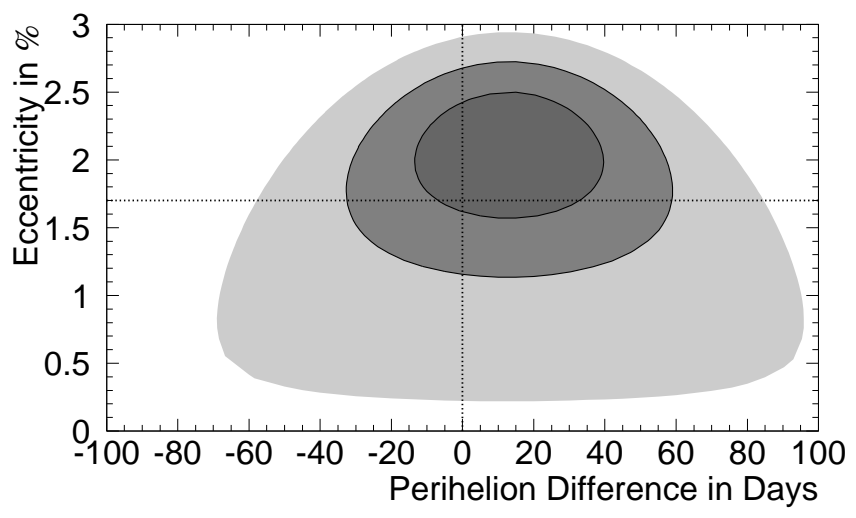

FIG. 1: Allowed Regions for the Eccentricity-Induced Seasonal Solar Neutrino Flux Variation at 68\% (dark gray), 95\% (gray), and $99.73 \%$ (light gray) C.L..

and the signal factor $s_{i \kappa}=p\left(\cos \theta_{i \kappa}, E_{\kappa}\right)$. The likelihood

$$
\mathcal{L}=e^{-\left(\sum_{i} B_{i}+S\right)} \prod_{i=1}^{N_{\mathrm{bin}}} \prod_{\kappa=1}^{n_{i}}\left(B_{i} \cdot b_{i \kappa}+S \frac{\mathrm{MC}_{i}}{\sum_{j} \mathrm{MC}_{j}} \cdot s_{i \kappa}\right)
$$

is maximized with respect to the signal $S$ and the 21 backgrounds $B_{i} . \mathrm{MC}_{i}$ is the number of events expected in energy bin $i$ using the flux and spectrum of ${ }^{8} \mathrm{~B}$ and hep neutrinos.

A simple determination of the day/night asymmetry is obtained by dividing the data sample into day and night and fit $\cos \theta_{\text {sun }}$ to each sample separately. From the obtained day (D) and night $(\mathrm{N})$ rates we calculate the asymmetry $A_{\mathrm{DN}}=(D-N) /(0.5(D+N))=$ $-2.1 \pm 2.0$ (stat) $)_{-1.2}^{+1.3}$ (syst) \% which is consistent with zero. To take into account time variations in the likelihood fit, the signal factors are modified to $s_{i \kappa}=p\left(\cos \theta_{i \kappa}, E_{\kappa}\right) \times$ $z_{i}\left(\alpha, t_{\kappa}\right)$ where $t_{\kappa}$ is the event time and $\alpha$ is an amplitude scaling factor. As a simple example, we measure the earth's orbital eccentricity. Since the neutrino flux is proportional to the inverse square of the distance between sun and earth, the eccentricity induces a seasonal time variation. Below $6.5 \mathrm{MeV}$ the background rates can fluctuate at time scales of several weeks or longer mainly due to changes in the radon contamination in water. Therefore, these energy bins are excluded from the eccentricity analysis by setting $z_{i}$ to 1 . To measure both the phase and amplitude of the variation, both the eccentricity and the perihelion is varied around the known values $(1.7 \%$ and $\sim$ January $3 \mathrm{rd}$ ). Figure 1 shows the allowed ranges of parameters at $68 \%, 95 \%$, and $99.73 \%$ C.L.. We measure the perihelion shift to be $13 \pm 17$ days (consistent with zero) and the amplitude of the neutrino flux variation to be $1.51 \pm 0.43$ (consistent with one) times the amplitude expected from $1.7 \%$ eccentricity.

To study neutrino oscillation-induced time variations, we correct for this seasonal effect (with the nominal 


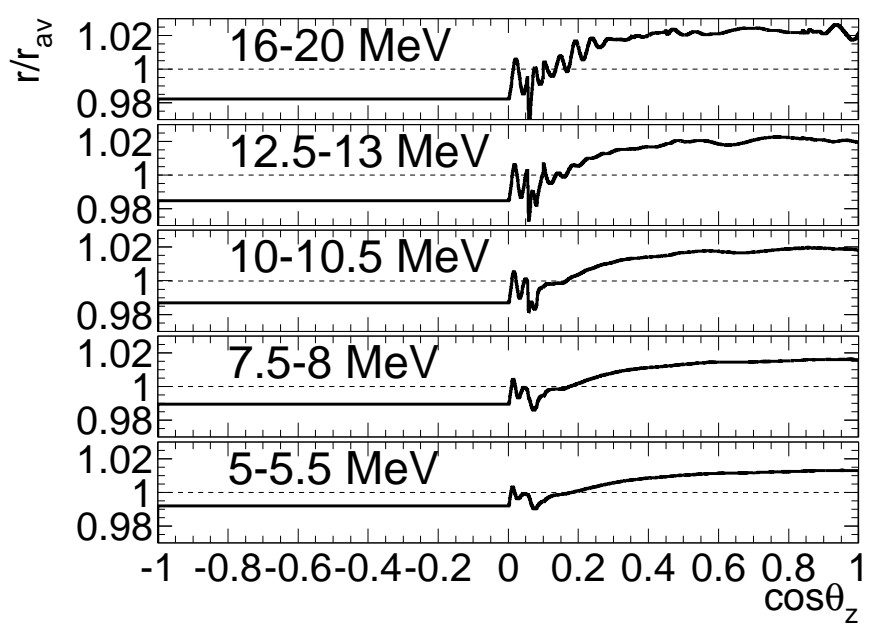

FIG. 2: LMA Solar Zenith Angle Variation Shapes. The predictions are for $\Delta m^{2}=6.3 \times 10^{-5} \mathrm{eV}^{2}$ in the energy bins 16 to $20 \mathrm{MeV}$ (top), 12.5 to $13 \mathrm{MeV}, 10$ to $10.5 \mathrm{MeV}, 7.5$ to 8 $\mathrm{MeV}$, and 5-5.5 MeV (bottom).

perihelion and eccentricity). After that, the additional seasonal amplitude variation is $0.48 \pm 0.43$ times the eccentricity-induced variation which is consistent with zero. We search for solar zenith angle variations (employing the solar zenith angle as the time variable) and additional seasonal variation due to the oscillation phase (using the distance between sun and earth). In each bin $i$ we calculate the rate $r_{i}(t)$ (oscillated Monte Carlo). From this rate and the live-time distribution the average $\left(r_{i}^{\text {av }}\right)$, day, and night rates and subsequently the day/night asymmetry $A_{i}$ are computed. Using the day (night) live-times $L_{D}\left(L_{N}\right)$ and the live-time asymmetry $L_{D N}=\left(L_{D}-L_{N}\right) /\left(0.5\left(L_{D}+L_{N}\right)\right)$, the effective asymmetry parameter $a_{i}=0.25 A_{i} L_{D N}$ is computed and $z_{i}(\alpha, t)$ is defined as

$$
z_{i}(\alpha, t)=\frac{1+\alpha\left(\left(1+a_{i}\right) r_{i}(t) / r_{i}^{\mathrm{av}}-1\right)}{1+\alpha \times a_{i}},
$$

so that $r_{i}^{\prime}(\alpha, t)=z_{i}(\alpha, t) \times r_{i}^{\text {av }}$ has the same average total rate $r_{i}^{\text {av }}$, but the day/night asymmetry is $A_{i} \times \alpha$. In particular, $r_{i}^{\prime}(0, t)=r_{i}^{\text {av }}$ is independent of $t$ and $r_{i}^{\prime}(1, t)=r_{i}(t)$. Figure 2 shows the expected solar zenith angle variation shapes $z_{i}\left(1, \cos \theta_{z}\right)$ in five different energy bins using an LMA solution and the density model of the earth [7].

The resulting likelihood function is maximized with respect to signal $S$, the backgrounds $B_{i}$, and the asymmetry scaling parameter $\alpha$. For the best-fit LMA oscillation parameters (which will be described later) we find $\alpha=0.86 \pm 0.77$ which corresponds to the day/night asymmetry

$$
A_{\mathrm{DN}}=-1.8 \pm 1.6(\text { stat })_{-1.2}^{+1.3}(\text { syst }) \%
$$

where $-2.1 \%$ is expected for these parameters. The statistical uncertainty is reduced by $25 \%$ with this likelihood

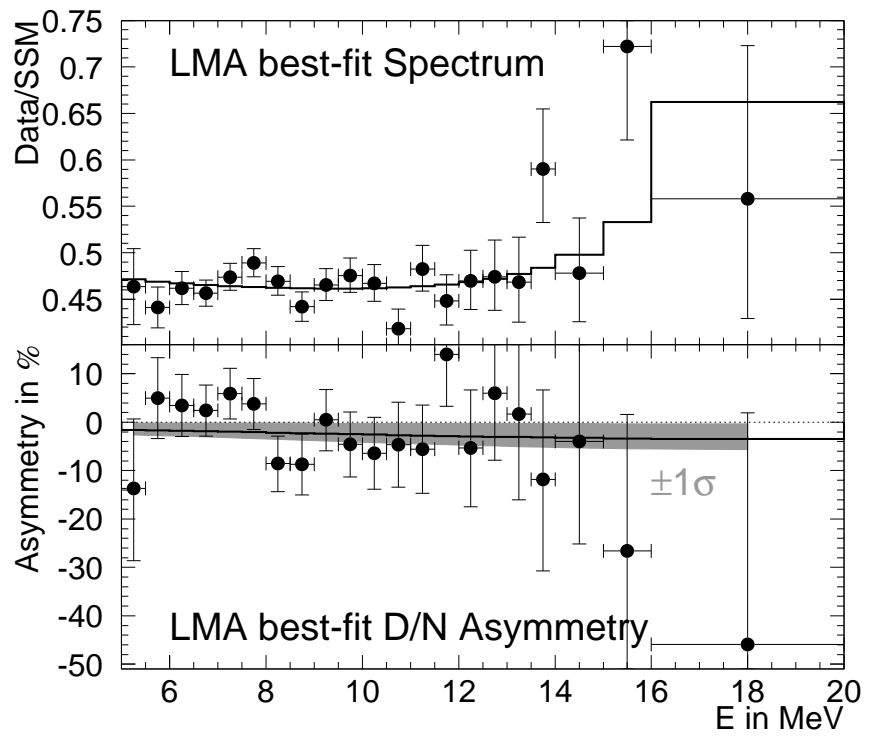

FIG. 3: LMA Spectrum (top) and D/N Asymmetry (bottom). The predictions (solid lines) are for $\tan ^{2} \theta=0.55$ and $\Delta m^{2}=$ $6.3 \times 10^{-5} \mathrm{eV}^{2}$ with $\phi_{8_{B}}=0.96 \times$ Standard Solar Model [8] and $\phi_{\text {hep }}=3.6 \times$ Standard Solar Model. Each energy bin is fit independently to the rate (top) and the day/night asymmetry (bottom). The gray bands are the $\pm 1 \sigma$ ranges corresponding to the fitted value over the entire range $5-20 \mathrm{MeV}: A=-1.8 \pm$ $1.6 \%$.

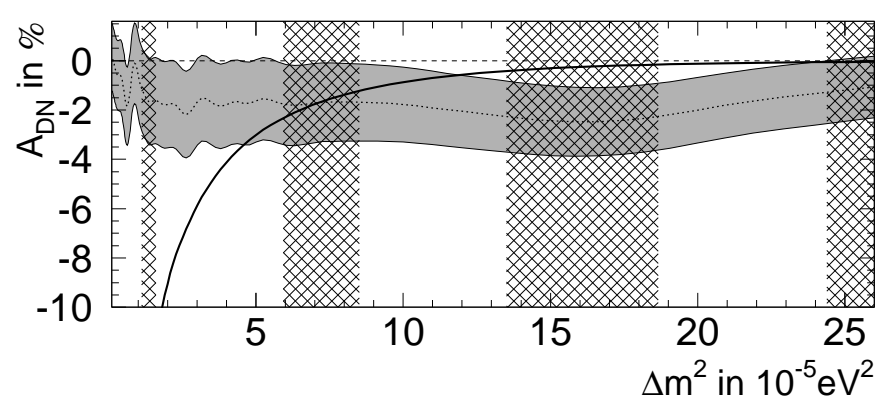

FIG. 4: SK Day/Night Asymmetry as a Function of $\Delta m^{2}$. The solid line is expected from two-neutrino oscillations, the band $( \pm 1 \sigma)$ results from the fit to the SK data. The mixing angle $\tan ^{2} \theta=0.55$ is used. Overlaid are the allowed ranges in $\Delta m^{2}$ (cross-hatched bands) from the KamLAND experiment [4]. The second band (LMA-I) is favored.

analysis; however, the resulting day/night asymmetry is still consistent with zero. Figure 3 shows the fitted rate (top), as well as the day/night asymmetry (bottom) for each energy bin separately. The oscillation expectations are indicated by the solid lines. The asymmetry fit value and uncertainty depends on the solar zenith angle variation shapes $z_{i}(1, t)$ which in turn depend on the oscillation parameters. Figure 4 shows the expected day/night asymmetry and fit results for each $\Delta m^{2}$ in the LMA region with the best-fit mixing angle $\tan ^{2} \theta=0.55$. The expected day/night asymmetry and the $\pm 1 \sigma$ band of the fit overlap between $5-12 \times 10^{-5} \mathrm{eV}^{2}$. 


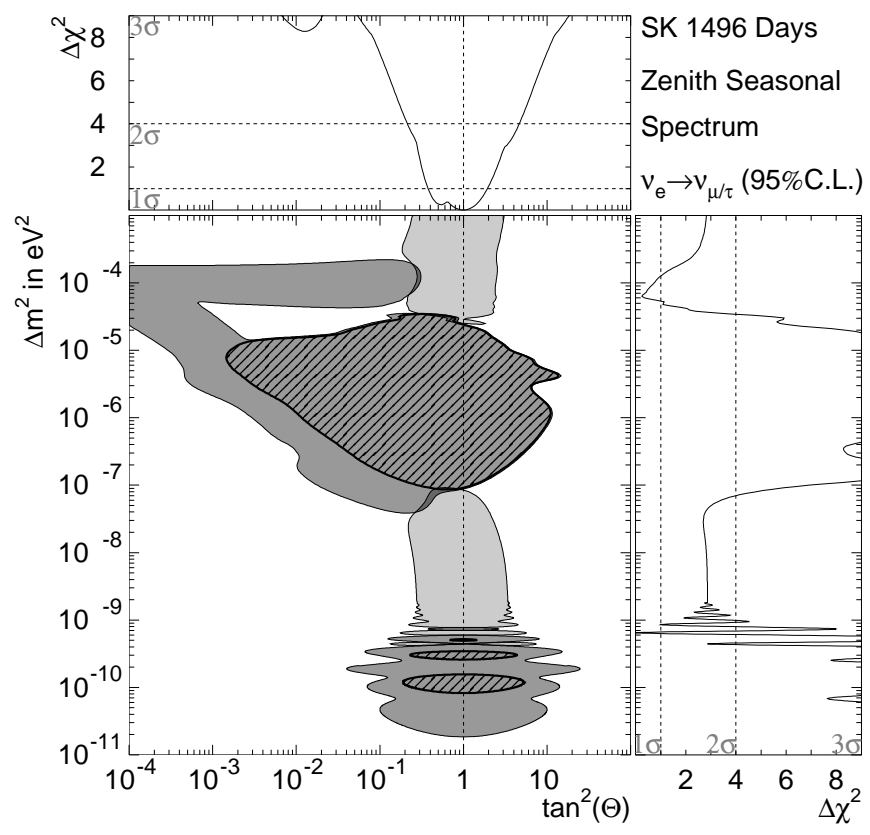

FIG. 5: Excluded (SK spectrum and time variation; dark gray) and Allowed (SK spectrum, rate, and time variation; light gray) at $95 \%$ C.L.. Overlaid are the areas excluded just by the day/night and seasonal variation (hatched regions inside thick black lines). The graphs at the top (and right) show the $\chi^{2}$ difference as a function of $\tan ^{2} \theta\left(\Delta m^{2}\right)$ alone where the $\Delta m^{2}\left(\tan ^{2} \theta\right)$ is chosen to minimize $\chi^{2}$.

To constrain neutrino oscillation using the SK rate time variations, the likelihood difference $\Delta \log \mathcal{L}=$ $\log \mathcal{L}(\alpha=1)-\log \mathcal{L}(\alpha=0)$ between the expected time variation and no time variation is computed. Below $\Delta m^{2}=1.8 \times 10^{-9} \mathrm{eV}^{2}$, the day $/$ night variation is replaced by an additional seasonal variation due to the oscillation phase. As for the eccentricity-induced variation, the energy bins below $6.5 \mathrm{MeV}$ are excluded from the seasonal variation because of the slow time variation of the background. However, since the effect of that variation on the day/night asymmetry was carefully evaluated to be negligible, these energy bins participate in the day/night variation. To combine the time variation constraints with those from the recoil electron spectrum, $\Delta \log \mathcal{L}$ is interpreted as a time-variation $\Delta \chi_{\mathrm{tv}}^{2}=-2 \Delta \log \mathcal{L}$ and added to the spectrum $\chi^{2}$.

Disregarding oscillations, we calculate the interaction rates in bin $i^{8} \mathrm{~B}_{i}\left(h^{2} p_{i}\right)$ due to ${ }^{8} \mathrm{~B}(h e p)$ neutrinos. We also compute the oscillated rates ${ }^{8} \mathrm{~B}_{i}^{\text {osc }}$ and hep ${ }_{i}^{\text {osc }}$ for each $\tan ^{2} \theta$ and $\Delta m^{2}$. From these and the measured rates $\operatorname{Data}_{i}$, we form the ratios $d_{i}=\operatorname{Data}_{i} /\left({ }^{8} \mathrm{~B}_{i}+\right.$ hep $\left._{i}\right)$, $b_{i}={ }^{8} \mathrm{~B}_{i}^{\text {osc }} /\left({ }^{8} \mathrm{~B}_{i}+\mathrm{hep}_{i}\right)$, and $h_{i}={ }^{8} \mathrm{hep}_{i}^{\text {osc }} /\left({ }^{8} \mathrm{~B}_{i}+\mathrm{hep}_{i}\right)$. The expected oscillation suppressions are $\beta b_{i}+\eta h_{i}$ where $\beta(\eta)$ is the ${ }^{8} \mathrm{~B}(h e p)$ neutrino flux scaling parameter. These suppressions are modified by the correlated uncertainty distortion functions $f_{i}^{B}\left(\delta_{B}\right)$ (uncertainty in the ${ }^{8} \mathrm{~B}$ neutrino spectrum), $f_{i}^{S}\left(\delta_{S}\right)$ (uncertainty in SK energy

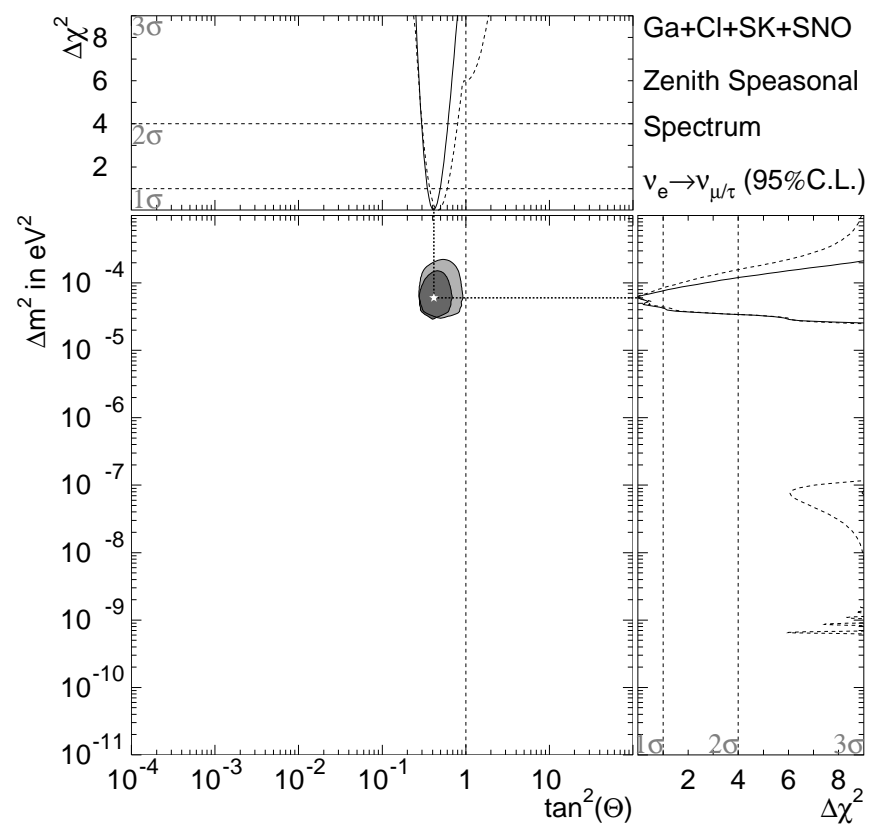

FIG. 6: Allowed area at 95\% C.L from the combination of SK and SNO (gray) and all solar data (dark gray). The graphs at the top (and right) show the $\chi^{2}$ difference as a function of $\tan ^{2} \theta\left(\Delta m^{2}\right)$ only: the dashed line is the SK/SNO fit, the solid line includes all solar data. The best fit to all solar data is $\tan ^{2} \theta=0.42$ and $\Delta m^{2}=6.0 \times 10^{-5} \mathrm{eV}^{2}$.

scale), and $f_{i}^{R}\left(\delta_{R}\right)$ (uncertainty in SK energy resolution) to $\rho_{i}=\frac{\beta b_{i}+\eta h_{i}}{f_{i}}$ where $f_{i}=f_{i}^{B} f_{i}^{R} f_{i}^{S}$. With the total energy bin-uncorrelated uncertainty $\sigma_{i}^{2}=\sigma_{i, \mathrm{stat}}^{2}+\sigma_{i, \mathrm{sys}, \mathrm{u}}^{2}$ the total $\chi^{2}$ is then

$\chi^{2}=\sum_{i=1}^{N_{\text {bin }}}\left(\frac{d_{i}-\rho_{i}}{\sigma_{i}}\right)^{2}+\frac{\delta_{B}^{2}}{\sigma_{B}^{2}}+\frac{\delta_{S}^{2}}{\sigma_{S}^{2}}+\frac{\delta_{R}^{2}}{\sigma_{R}^{2}}+\Delta \chi_{\mathrm{tV}}^{2}+\left(\frac{\beta-1}{\sigma_{f}}\right)^{2}$

where the last term constraining the ${ }^{8} \mathrm{~B}$ flux to the standard solar model (SSM) [8] is optional. Including this last term, the best oscillation fit is in the quasi-vacuum region at $\Delta m^{2}=6.49 \times 10^{-10} \mathrm{eV}^{2}$ and maximal mixing, where a summer/winter asymmetry of $-0.6 \%$ is expected and $-0.3 \pm 0.7 \%$ (stat) is found. The $\chi^{2}$ is 17.1 for 20 degrees of freedom $(65 \%$ C.L.). The LMA solution fits almost equally well: the smallest $\chi^{2}$ at $\Delta m^{2}=6.3 \times 10^{-5} \mathrm{eV}^{2}$ and $\tan ^{2} \theta=0.55$ is 17.3 (63\% C.L.). Figure 5 shows the allowed areas at $95 \%$ C.L. using all SK information: rate, spectrum and time-variation. It also shows the $\Delta \chi^{2}$ as a function of $\tan ^{2} \theta\left(\Delta m^{2}\right)$ alone: SK data excludes small mixing at more than $3 \sigma$. SK data also disfavors $\Delta m^{2}>10^{-3} \mathrm{eV}^{2}$ and $2 \times 10^{-9} \mathrm{eV}^{2}<\Delta m^{2}<3 \times 10^{-5} \mathrm{eV}^{2}$.

Stronger constraints on $\Delta m^{2}$ result from the combination of SK with other solar neutrino data [1]. The combined fit to SK data and the SNO measurements on the charged-current and neutral-current reactions of solar ${ }^{8} \mathrm{~B}$ neutrinos with deuterons need not constrain any neutrino flux with a solar model. Figure [6] shows the 

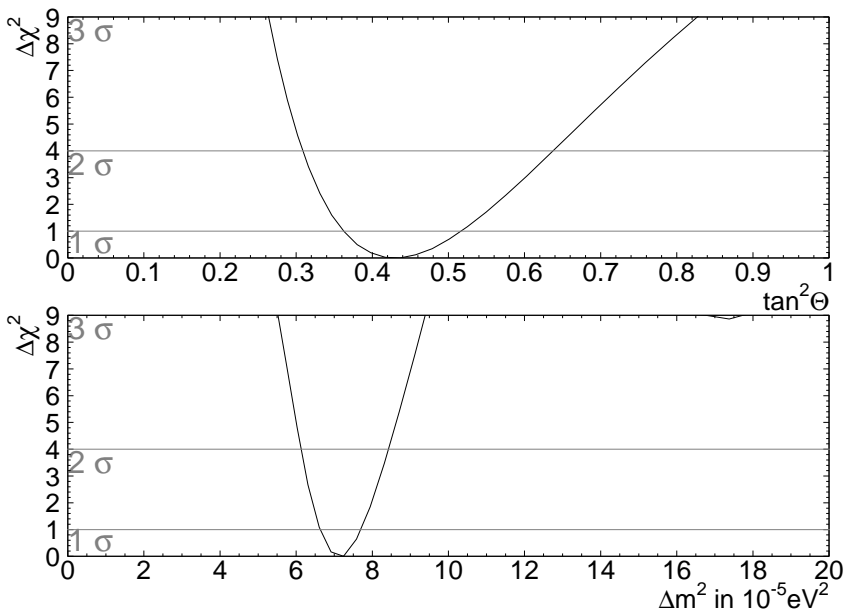

FIG. 7: $\Delta \chi^{2}$ Curves as a Function of Mixing (Top) and $\Delta m^{2}$ (Bottom) Using all Solar and KamLAND Data. Only LMA-I remains allowed at $3 \sigma$.

allowed region at 95\%C.L.: only LMA solutions survive. When the charged-current rates measured by Homestake, GALLEX/GNO, and SAGE are included as well, the LMA solutions are favored by $3 \sigma$; however, the fit relies on the SSM predictions of the pp, pep, CNO, and ${ }^{7}$ Be neutrino fluxes.

The first oscillation analysis of the KamLAND reactor neutrino spectrum and rate leaves several allowed areas, usually called LMA-0, LMA-I, LMA-II, and LMA-III. When we combine the analysis of SK with all other solar experiments and a likelihood analysis of the KamLAND data [9], the LMA-I is strongly favored over the other solutions: in Figure 7 the $\Delta \chi^{2}$ of the fit is plotted against $\Delta m^{2}$ and $\tan ^{2} \theta$.

In summary, SK has measured very precisely the ${ }^{8} \mathrm{~B}$ neutrino flux time-variations expected from twoneutrino oscillations. For the best LMA parameters, the day/night asymmetry is determined as $=-1.8 \pm$ 1.6 (stat) ${ }_{-1.2}^{+1.3}$ (syst) $\%$ where $-2.1 \%$ is expected. SK data disfavor large $\Delta m^{2}$ LMA solutions, since their expected day/night asymmetries are closer to zero. In combination with other solar data and the KamLAND reactor neutrino results, the oscillation parameters are determined as $\Delta m^{2}=7.1_{-0.5}^{+0.6} \times 10^{-5} \mathrm{eV}^{2}$ and $\tan ^{2} \theta=0.44 \pm 0.08$.

The authors acknowledge the cooperation of the Kamioka Mining and Smelting Company. The SuperKamiokande detector has been built and operated from funding by the Japanese Ministry of Education, Culture, Sports, Science and Technology; the U.S. Department of Energy; and the U.S. National Science Foundation. This work was partially supported by the Korean Research Foundation (BK21) and the Korea Ministry of Science and Technology.
[*] Present address: Harvard University, Cambridge, MA 02138, USA

[ $\dagger$ ] Present address: The Institute of Physical and Chemical Reasearch (RIKEN), Wako, Saitama 351-0198, Japan

[ $\ddagger$ ] Present address: Department of Physics, University of Utah, Salt Lake City, UT 84112, USA

[1] B.T.Cleveland et al., Astrophys. J. 496, 505 (1998); V.Gavrin, Nucl. Phys. B (Proc. Suppl.) 118, 39 (2003); T.Kirsten, Nucl. Phys. B (Proc. Suppl.) 118, 33 (2003); S.Fukuda et al., Phys. Rev. Lett. 86, 5656 (2001); Q.R.Ahmad et al., Phys. Rev. Lett. 89, 011301 (2002); Q.R.Ahmad et al., Phys. Rev. Lett. 89, 011302 (2002).

[2] S.Fukuda et al., Phys. Rev. Lett. 86, 5651 (2001).
[3] S.Fukuda et al., Phys. Lett. B 539, 179 (2002).

[4] K.Eguchi et al., Phys. Rev. Lett. 90, 021802 (2003).

[5] S.P.Mikheyev and A.Y.Smirnov, Sov. Jour. Nucl. Phys. 42, 913 (1985); L.Wolfenstein, Phys. Rev. D 17, 2369 (1978).

[6] S.Fukuda et al., Nucl. Instrum. Methods A 501, 418 (2003).

[7] A.M.Dziewonski and D.L.Anderson, Phys. Earth Planet. Inter. 25, 297 (1981).

[8] J.N.Bahcall et al., Astrophys. J. 555, 990 (2001).

[9] A.Ianni, hep-ph/0302230v2; to be published in Journal of Physics $G$ 